\title{
An Interesting Case of Early Multifocal Gastric Cancer
}

\author{
Onur Keskin, Necati Ormeci
}

\begin{abstract}
Early gastric cancer (EGC) is defined as gastric cancer confined to mucosa or submucosa, regardless of the presence or absence of lymph node metastasis. Generally, patients with multifocal EGC have two or three lesions but rarely have more than three lesions. In this article, we present a patient who was admitted to the hospital with complaints of epigastric pain and fatique. His endoscopic examination revealed four EGC lesions. We performed endoscopic mucosectomy for all lesions successfully. Endoscopic mucosal resection is an organ conservative approach and can be performed to patients with early multifocal gastric cancer. During this procedure life-threatening severe complications can occur. In our experience, severe bleeding occured during mucosectomy and our patient underwent subtotal gastrectomy.
\end{abstract}

Abbreviation: EGC: Early gastric cancer.

Keywords: Early gastric cancer, Multifocal, Endoscopic mucosal resection, Complications.

How to cite this article: Keskin O, Ormeci N. An Interesting Case of Early Multifocal Gastric Cancer. Euroasian J HepatoGastroenterol 2012;2(2):116-118.

Source of support: Nil

Conflict of interest: None

\section{INTRODUCTION}

Early gastric cancer (EGC) is defined as gastric cancer confined to mucosa or submucosa, regardless of the presence or absence of lymph node metastasis. The diagnosis and recognition of EGC has been increasing with a favorable prognosis (5-year survival rate of over 90\% following surgery). ${ }^{1}$

Multiple gastric cancer is not a rare condition. Multifocal gastric carcinoma was reported with an incidence of 5.1\% and more than half of multifocal gastric carcinomas were at an early stage. Generally, patients with multifocal gastric cancer have two or three lesions but rarely have more than three lesions. ${ }^{2}$ As compared to advanced cancer, higher incidence of multifocal lesions in EGC have been demostrated. ${ }^{3,4}$

An inverse correlation is found between tumor size and multifocality. Most patients with multifocal early gastric cancer, size of their lesions frequently is less than $10 \mathrm{~mm}$ in their greatest diameter. Nearly 20 to $30 \%$ of accessory lesions are likely to be missed in preoperative assessments. ${ }^{5}$

Macroscopically, multifocal carcinomas often are flat and small, rarely infiltrating submucosa; and mostly both primary and secondary lesions were of the same histologic type. No significant difference in postoperative survival was demonstrated between patients with multifocal EGC and solitary EGC. ${ }^{2}$

Multifocal gastric carcinoma is more frequent when patients are older and tumors are small and early. Therefore, lesions are likely to be missed in preoperative or intraoperative diagnostic assessment. In such cases, entire stomach should be examined by particular care before and during the operation, especially when local or endoscopic resection of the tumor is to be performed. In this article, we present a patient with four EGCs, type I, who had severe hemorrhage during endoscopic polipectomy.

\section{CASE REPORT}

An 80-year-old man was admitted to the hospital with complaints of epigastric pain, fatigue and weakness. He had no history of bleeding, weight loss, nausea and vomiting. There were no abnormal findings on physical examination.

In his endoscopic examination, there was a long segment Barrett esophagitis between 38 and 42th $\mathrm{cm}$ of esophagus. Besides that there were also multifocal tumoral lesions located at proximal and anterior part of corpus $4 \mathrm{~cm}$ in diameter, proximal and posterior wall of corpus, $3 \mathrm{~cm}$ in diameter, at distal part of corpus greater curvature side $8 \mathrm{~mm}$ in diameter, posterior wall of corpus $10 \mathrm{~mm}$ in diameter (Figs 1A to C). These lesions were soft, sessile and had villous type polypoid characteristics. There were no ulcerated lesions, erosions or active scars in the stomach.

Endoscopic biopsies taken from those lesions revealed early gastric adenocarcinoma invaded to mucosa. The patient persistently refused to have surgical operation for

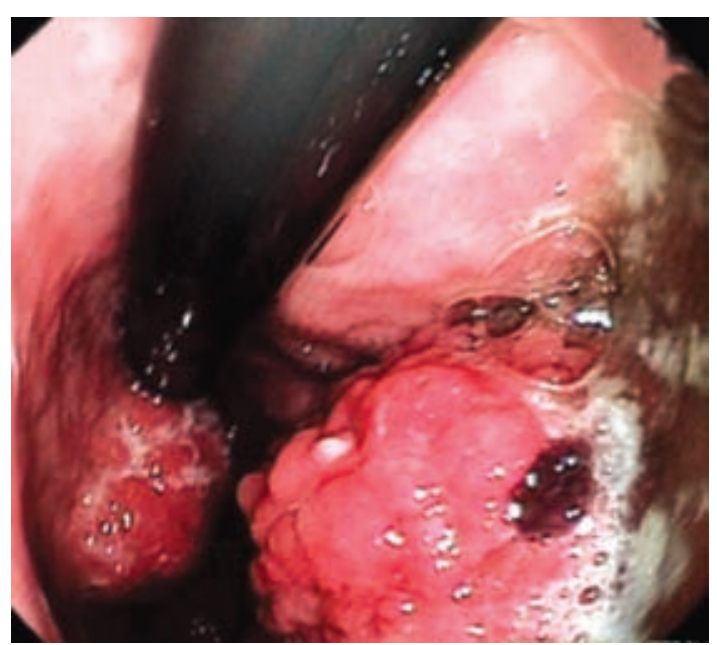

Fig. 1A: Two polypoid lesions at proximal and anterior part of corpus $-4 \mathrm{~cm}$ in diameter, proximal and posterior wall of corpus$3 \mathrm{~cm}$ in diameter 


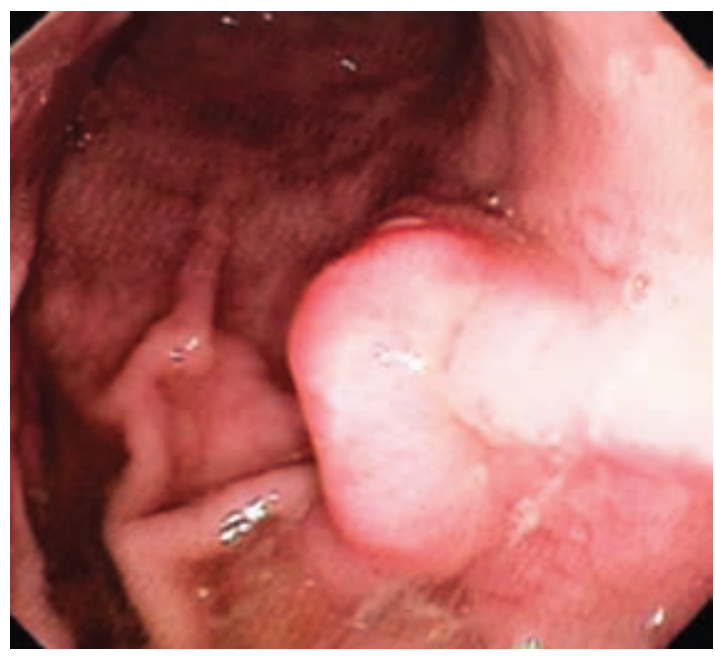

Fig. 1B: A polypoid lesion located at the posterior wall of corpus $-10 \mathrm{~mm}$ in diameter

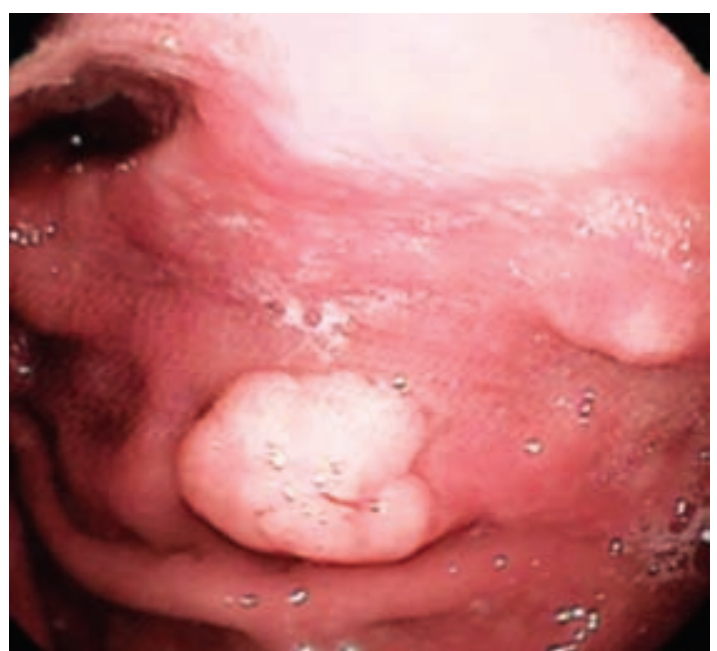

Fig. 1C: An elavated lesion located at distal part of corpus greater curvature side $-8 \mathrm{~mm}$ in diameter

gastric cancer. Endoscopic resection was performed for all lesions. Three of them were succesfully resected but just after resection of fourth lesion, which was located at the proximal corpus of anterior wall and $4 \mathrm{~cm}$ in diameter, severe arterial bleeding occured at the base of polyp in three different focus. Although we have used argon laser coagulator, the patient had shock in a few minutes. The patient underwent emergency subtotal gastrectomy. Pathologic examination of the gastrectomy material revealed lesions concordant with multifocal intramucosal early gastric cancer without lymph node metastasis (Figs 2A and B).

Fortunately he had no complication during operation had no serious problem in postoperative period.

\section{DISCUSSION}

Because early multifocal gastric cancer is not a rare condition, we must evaluate all mucosal surfaces and be very careful for presence of other lesions during endoscopic

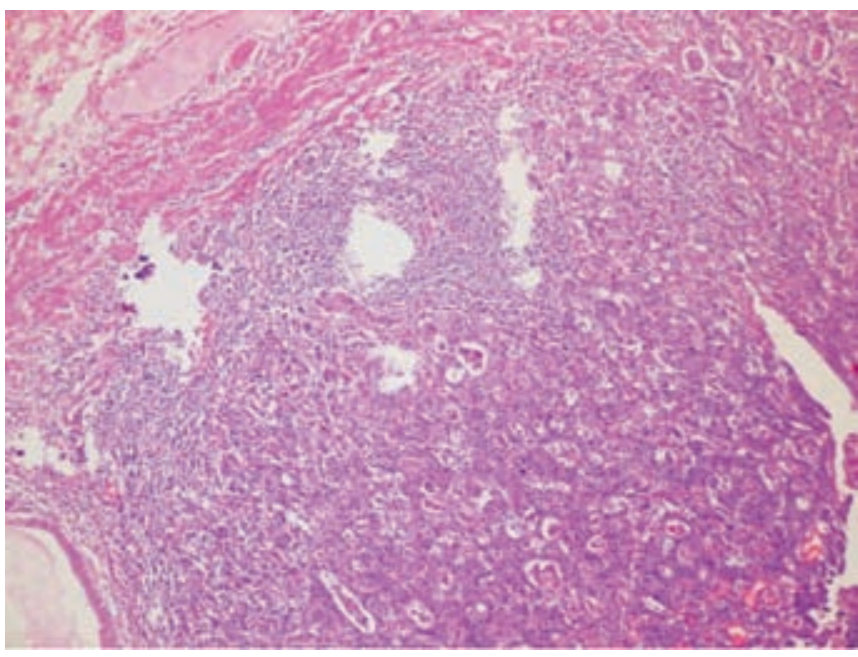

Fig. 2A: Early gastric cancer which is confined to mucosa and associated with an adenomatous polyp. This low microscopic power view also shows areas of intestinal metaplasia and atrophic gastritis

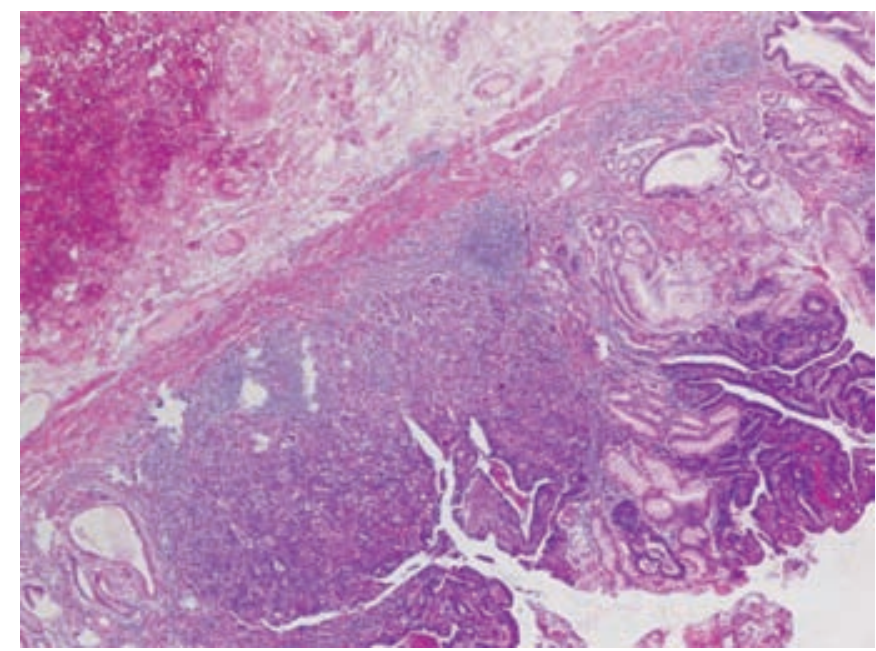

Fig. 2B: Early gastric cancer which is associated with tubulovillous adenoma

examination. EGC can be treated endoscopically. ${ }^{6}$ Endoscopic mucosectomy can also be performed to multifocal cancers but severe complications can occur during endoscopic mucosal resention procedures. Bleeding was reported in 1 to $20 \%$ in different studies ${ }^{7}$ and incidence of perforation was detected in $1.2 \%$ in a Japanase study. ${ }^{8}$ These complications can also be succesfully treated endoscopically. Advancements in endoscopic technologies provided us not only to diagnose stomach cancer in early stage but also to notice early recurrence. Acccording to the literature the patients who have mucosal cancer, welldifferantiated adenocarcinoma, less than $2 \mathrm{~cm}$ in diameter and without lymph node metastasis have favorable prognosis without recurrences. ${ }^{8}$

For this patient, we performed endoscopic resection for all lesions successfully. But after resection of fourth lesion, severe arterial bleeding occured. Finally, patient underwent emergent subtotal gastrectomy. 


\section{CONCLUSION}

We should give efforts for the diagnosis of stomach cancer in early stages. We must be aware of multifocal stomach cancer during endoscopic examination. Because this approach is organ conservative, endoscopic mucosectomy should be preferred.

\section{REFERENCES}

1. Borie F, Plaisant N, Millat B, Hay M, Fagniez PL, Saxce BD. Treatment and prognosis of early multiple gastric cancer. Eur J Surg Oncol 2003;29:511-14.

2. Otsuji E, Kurui Y, Ichikava D, Okamoto K, Hagiwara A, Yamagishi H. Clinicopathologic characteristic and prognosis of synchronous multifocal gastric carcinomas. Am J Surg 2005; 189:116-19.

3. Noguchi Y, Ohta H, Tagaki K, et al. Synchronous multiple early gastric carcinoma: A study of 178 cases. World J Surg 1995;9: 786-93.

4. Kitamura K, Yamaguchi T, Okamoto K, et al. Clinicopathologic features of synchronous multifocal early gastric cancers. Anticancer Res 1997;17;643-46.
5. Mitsudomi T, Watanabe A, Matsusaka T, Fujinaga Y, Fuchigami T, Iwashita A. A clinicopathological study of synchronous multiple gastric cancer. Br J Surg 1989;76:237-40.

6. Kojima T, Parro-Blanco A, Takahashi H, Fujita R. Outcome of endoscopic mucosal resection for early gastric cancer: Review of Japanese literature. Gastrointest Endosc 1998;48:550-54.

7. Marc G, Lopes CV. Endoscopic resection of superficial gastrointestinal tumors. World J Gastroenterol 2008;14;4600-06.

8. Oda Ichiro, Saito D, Tada M, et al. A multicenter retrospective study of endoscopic resection for early gastric cancer. Gastric Cancer 2006;9:262-70.

\section{ABOUT THE AUTHORS}

\section{Onur Keskin (Corresponding Author)}

Department of Gastroenterology, Ibni Sina Hospital, Ankara University School of Medicine, Samanpazari, Ankara, Turkey, Phone: 031250821 66, e-mail: onur_keskin@yahoo.com

\section{Necati Ormeci}

Department of Gastroenterology, Ankara University School of Medicine, Ankara, Turkey 